4

7

8

9

10

11

12

\title{
Hot melt extruded zein for controlled delivery of diclofenac sodium: effect of drug
} loading and medium composition
Lorina Bisharat $^{\mathrm{a}^{*}}$, Hatim S. Alkhatib ${ }^{\mathrm{a}}$, Amer Abdelhafez ${ }^{\mathrm{a}}$, Aya Barqawi ${ }^{\mathrm{a}}$, Ahmad Aljaberi ${ }^{\mathrm{b}}$, Sheng Qi ${ }^{\mathrm{c}}$, Alberto Berardi ${ }^{\mathrm{b}}$

a Department of Pharmaceutics and Pharmaceutical Technology, School of Pharmacy, The University of Jordan, Amman 11942, Jordan.

b Department of Pharmaceutical Sciences and Pharmaceutics, Faculty of Pharmacy, Applied 3 Science Private University, Amman 11931, Jordan.

c School of Pharmacy, University of East Anglia, Norwich NR7 1AT, UK.

* Corresponding author: Department of Pharmaceutics and Pharmaceutical Technology, School of Pharmacy, The University of Jordan, Amman 11942, Jordan. Email: 17 L.bisharat@ju.edu.jo, Tel. +9626 5355000; Fax: +962 65300250. 
31 This study evaluates the potential use of zein as an excipient in hot-melt extrusion for controlled delivery of diclofenac sodium (DS). Mixtures of zein, polyethylene glycol and 33 drug were hot melt extruded and cut into $2 \mathrm{~mm}$ extrudates. Extrudates were characterised using differential scanning calorimetry, X-ray powder diffraction and scanning electron microscopy. The drug in the extrudates was found to be in the non-crystalline state, independent of the drug loading. Moreover, the drug release from extrudates was investigated. The release was directly dependent on the drug loading: a controlled and nearly zero-order release was obtained at the lowest drug loading $(12.5 \% \mathrm{w} / \mathrm{w})$, whereas almost immediate release was achieved at higher drug loadings, i.e. $25 \%$ and $37.5 \%$. The release was inversely dependent on the ionic strength of the medium. The influence of digestive enzymes on drug release was also studied. Pancreatin, but not pepsin, was found to have a significant influence on the drug release as well as on the microstructure of zein extrudates. These data therefore support the potential use of zein as excipient in hot melt extrusion for controlled 44 release purposes.

Keywords: Zein, Hot melt extrusion, Controlled release, Solid dispersion, Amorphous, 


\section{Introduction}

57

58 Controlled-release oral dosage forms are designed to achieve drug release characteristics over

a specific time course that can offer certain therapeutic objectives over conventional dosage forms. Polymeric excipients have been used in oral formulations to control drug release rate. Although synthetic and semisynthetic polymers have been extensively used for this purpose, natural excipients, being both sustainable and biodegradable, are being increasingly used. Zein, for example, is a plant-derived protein, that has been extensively studied for its potential use as an excipient in the pharmaceutical industry (Berardi et al., 2018).

Zein is the major storage protein of corn (Shukla and Cheryan, 2001), and constitutes 44-79\% of the endospermic proteins (Lawton, 2002). It is composed of a mixture of different peptides that can be classified on the basis of their solubility and molecular weight into $\alpha$ (19 and $22 \mathrm{kDa}), \beta(17-18 \mathrm{kDa}), \gamma(16$ and $27 \mathrm{kDa})$ and $\delta(10 \mathrm{kDa})$ zeins (Esen, 1986). Zein is particularly rich in hydrophobic amino acids, but deficient in polar or ionisable amino acids (Cabra et al., 2006; Shukla and Cheryan, 2001). Zein is a natural, biocompatible and biodegradable material produced from sustainable sources (Berardi et al., 2018). Due to its amino acid composition, zein is insoluble in water, yet is soluble in aqueous ethanol solutions. Despite its insolubility in water, zein tends to swell in the presence of aqueous medium (Beck et al., 1996). This is because zein has hydrophilic regions in addition to the hydrophobic domains, and thus behaves as an amphiphilic protein (Wang et al., 2008). Indeed, zein matrices were found to swell in a hydrophilic-like fashion, but were nonerodible as hydrophobic matrices (Berardi et al., 2017b). Being swellable, yet insoluble and nonerodable, zein has been also studied for its potential use as an excipient for controlled drug release in nano- and microscale as well as macroscale formats. Zein tablets (Berardi et 
81 al., 2017a; Georget et al., 2008; Katayama and Kanke, 1992; Li et al., 2010; O’Donnell et al., 1997; Raza et al., 2020, 2019) and capsules (Berardi et al., 2017b) were successfully developed for controlled release purposes. However, the potential use of zein biopolymer as an excipient for direct compression could be limited by its poor flowability and tabletability, compared to the commonly used excipients, as suggested by Berardi et al. (Berardi et al., 2017a). Similarly, Georget et al. (Georget et al., 2008) reported that tablets core were striated in appearance, due to the elastic recovery of zein upon removal of the compression force.

Hot melt extrusion (HME) technique involves forcing raw materials using a rotating screw(s) through a die of a defined geometry under elevated temperature (Crowley et al., 2007). HME is a continuous and solvent free process, reducing the number of steps and eliminating the drying step (Crowley et al., 2007). Recently, Zein has proved to be a promising new excipient for HME for the development of controlled drug delivery systems (Bouman et al., 2016, 2015). Despite being a protein, it has been shown that zein is relatively heat stable and can be extruded at high temperatures (up to $160{ }^{\circ} \mathrm{C}$ ) without significant changes in its molecular or physical properties (Selling, 2010). Zein and paracetamol physical mixtures were hot-melt extruded injection moulded into single-matrix phase, homogenous caplets by Bouman et al. (Bouman et al., 2015). Controlled drug release was obtained and was found to be strongly dependent on device dimensions but nearly independent of drug loading. In another work,

100 Bouman et al. studied the influence of drug hydrophobicity as well as the influence of 101 electrostatic interactions between drug and polymer, which is governed by $\mathrm{pH}$ medium, on 102 drug release (Bouman et al., 2016). They found out that the release kinetics were not mainly determined by water solubility of the drug, yet the electrostatic attractions between zein matrix and drug can significantly slow down the release. 
106 In this work, we developed controlled release zein matrices of diclofenac sodium (DS) using

107 hot melt extrusion. DS is a BCS class II drug that is insoluble at acidic pH, yet soluble at

108 more basic intestinal pH (Chuasuwan et al., 2009), justifying its widespread use in controlled

109 release dosage forms (Jantratid et al., 2009; Rani and Mishra, 2004). The physical state of the

110 model drug was characterised by differential scanning calorimetry (DSC) and X-ray powder

111 diffraction (XRPD). Dissolution studies were performed to investigate the effect of drug

112 loading and composition of dissolution medium on drug release. The influence of the medium

113 ionic strength on drug release from zein extrudates was evaluated here for the first time. We

114 also studied the influence of $\mathrm{pH}$ switch, i.e. $\mathrm{pH} 1.2$ to $\mathrm{pH} 7.5$, and that of the presence of

115 digestive enzymes on the drug release and on the microstructure of zein extrudates. The

116 objective of this article is therefore to gain an understanding of what are the key factors that

117 might influence the performance of zein extrudates in conditions simulating the

118 heterogeneous environment of the upper gastrointestinal tract, and could ultimately dictate

119 zein's applications.

120

2. Materials and Methods

122

2.1 Materials

123

124 Zein from maize (Z3625) was obtained from Sigma-Aldrich (Germany) and used as received.

125 This product was plasticized with polyethylene glycol 400, PEG 400, obtained from Merck-

126 Schuchardt (Germany). The model drug, diclofenac sodium was obtained from Aarti drugs

127 Ltd. (India) and was used as received. Diclofenac sodium was chosen on the basis that an

128 extended release formulation of this, some are already available in market, can prove useful

129 in management of signs and symptoms of patients with osteoarthritis and rheumatoid

130 arthritis. Pepsin (p7125) and pancreatin (p8096) enzymes were purchased from Sigma- 
131 Aldrich (USA). Other materials used for the preparation of the dissolution media were reagents of standard grades and were supplied by AZ chem and Alpha Chemika (India).

\subsubsection{Hot melt extrusion}

137 Zein powder was first granulated in a wet granulation step in order to improve its flow

138 properties. PEG 400, added as an aqueous solution during granulation, was used to lower the 139 glass transition temperature (Tg) of zein. Zein and PEG 400 (10 or $20 \%$ of the total weight)

140 were mixed using a mortar and pestle until a suitable granule was formed. At concentrations

141 greater than $20 \%$ PEG, the granulation process was not possible. The wet granulation was

142 sieved through a $1 \mathrm{~mm}$ sieve, and then dried in oven at $50{ }^{\circ} \mathrm{C}$ overnight. The dried granules

143 were sieved again through the same sieve before being stored in a plastic bottle. The residual

144 moisture content was measured by the "loss on drying" (LOD) in triplicate using a moisture

145 balance (Mettler PE 160, USA). The LOD of granules containing 10\% and 20\% PEG 400

146 was $3.75 \%( \pm 0.78)$ and $3.98 \%( \pm 0.32)$, respectively.

148 Blends of pre-plasticized zein granules and different amounts of diclofenac sodium were 149 prepared to obtain formulations with drug loading ranging from $12.5 \%$ to $37.5 \%(\mathrm{w} / \mathrm{w})$. The

150 mixtures were hot-melt extruded using a single-screw extruder (Randcastle microtruder, 151 USA) (Andrews et al., 2008; Young et al., 2002), fitted with a cylindrical die with an inner 152 diameter of $5 \mathrm{~mm}$. The extrusions were performed at a temperature of $140{ }^{\circ} \mathrm{C}$ for all zones, 153 i.e. zone 1, 2,3 and 4, and a screw speed of $20 \mathrm{rpm}$. Extrudates were allowed to cool down and then stored in sealed containers at room conditions. 
156 The composition of zein extrudates is reported in Table 1. A special notation (e.g.,

157 E_12.5_20) was used to describe the preparation conditions: the letter E stands for extrudate,

158 the first number is the concentration of diclofenac sodium $(\mathrm{w} / \mathrm{w} \%)$ and the second number is

159 the plasticizer level (w/w\%) with respect to the total weight of zein and PEG 400.

160

161

\subsubsection{Drug Content}

162 Extrudates were ground using mortar and pestle. An amount equivalent to $25 \mathrm{mg}$ of

163 diclofenac sodium was weighed and transferred to a $100 \mathrm{ml}$ volumetric flask and phosphate

164 buffer $\mathrm{pH} 7.5$ was added. The flask was stirred for 1 hour using a magnetic bar and was then

165 sonicated in a bath sonicator for another 1 hour with intermittent shaking. The volume was

166 made to $100 \mathrm{~mL}$ and a $10 \mathrm{~mL}$ sample was taken and filtered using $0.45 \mu \mathrm{m}$ PTFE filters. The

167 samples were analysed for diclofenac sodium by high-performance liquid chromatography

168 (HPLC; Knauer, Leeds, UK). The mobile phase consisted of water- methanol (30:70 v/v)

169 adjusted to $\mathrm{pH} 3.5$ with glacial acetic acid and pumped at $1 \mathrm{~mL} \cdot \mathrm{min}^{-1}$ through a $\mathrm{C} 18,5 \mu \mathrm{m}$,

$170250 \times 4.6 \mathrm{~mm}$ column (Thermo Scientific, USA). UV detection was carried out at $276 \mathrm{~nm}$.

171 The measurements were performed in triplicate and the averages and standard deviations were calculated. The drug content of zein extrudates is reported in Table 1.

\subsubsection{Differential Scanning Calorimetry (DSC)}

DSC analysis of diclofenac sodium (DS) raw powder, zein raw powder, physical mixtures of

176 DS and zein powder or granulated zein as well as DS loaded zein extrudates was carried out

177 using DSC 250 (TA Instruments, USA). Approximately $5 \mathrm{mg}$ samples were sealed in standard aluminium DSC pans. Extrudates were crushed using mortar and pestle before measurements. The samples were heated from $25^{\circ} \mathrm{C}$ to $350^{\circ} \mathrm{C}$ at a heating rate of $10^{\circ} \mathrm{C} / \mathrm{min}$, under nitrogen purge $(50 \mathrm{ml} / \mathrm{min})$. 
183 X-Ray Powder Diffraction analysis of diclofenac sodium (DS) raw powder, zein raw powder, physical mixtures of DS and zein powder or granulated zein as well as DS loaded zein extrudates was carried out using XRD-7000 X-Ray Diffractometer (Shimadzu, Japan). The diffraction pattern was recorded using monochromatic $\mathrm{Cu}$ radiation $\left(\lambda=1.54 \mathrm{~A}^{\circ}\right)$ as anode material and operated at a voltage of $40 \mathrm{kV}$ and a current of $30 \mathrm{~mA}$. The samples were mounted on Al sample holder and XRD patterns were recorded in the range of $2-60^{\circ}$ at the speed of $2^{\circ} / \mathrm{min}$ and a sampling pitch of $0.02^{\circ}$.

\subsubsection{Dissolution Studies}

192

Dissolution studies were carried out at $37 \pm 0.5^{\circ} \mathrm{C}$ in a BP dissolution apparatus I (ERWEKA DT 600, ERWEKA GmbH, Germany), with a rotation speed of $100 \mathrm{rpm}$. Extrudates were manually cut into pieces of $2 \mathrm{~mm}$ long using a cutting blade. An appropriate amount of the previously cut extrudates $(2 \mathrm{~mm}$ ) of each formulation (Table 1), equivalent to $25 \mathrm{mg}$ drug, was weighed and placed in the basket. The tests were performed in $50 \mathrm{mM}$ phosphate buffer ( $\mathrm{pH}$ 7.5), as recommended by USP monograph for diclofenac sodium extended release tablets, or otherwise in $900 \mathrm{~mL}$ of water; $100 \mathrm{mM}$ phosphate buffer $(\mathrm{pH} 7.5) ; 200 \mathrm{mM}$ phosphate buffer $(\mathrm{pH} 7.5) ; 50 \mathrm{mM}$ phosphate buffer $(\mathrm{pH} \mathrm{7.5)}+0.1 \mathrm{M} \mathrm{NaCl} ; 50 \mathrm{mM}$

200 phosphate buffer $(\mathrm{pH} 7.5)+0.2 \mathrm{M} \mathrm{NaCl}$. Ten $\mathrm{mL}$ samples were withdrawn at predetermined times using a syringe, filtered through a $0.45 \mu \mathrm{m}$ PTFE membrane filter (Macherey-Nagel GmbH \& Co. KG, Germany) and replaced with an equivalent volume of fresh medium. The diclofenac sodium concentration was determined using a UV-VIS spectrophotometer 
appropriate standard curve. The dissolution studies were carried out in triplicate and the average drug release (calculated based on actual drug content) \pm SD was calculated.

208 Dissolution studies under conditions that more closely resemble those in the upper gastrointestinal tract were also carried out. In this case, freshly prepared simulated gastric

210 fluid (SGF) $\left(2 \mathrm{~g} \mathrm{~L}^{-1} \mathrm{NaCl}\right.$ and $3.2 \mathrm{~g} \mathrm{~L}^{-1}$ pepsin) $\mathrm{pH} 1.2$ was used as the dissolution medium

211 for the first 2 hours. This medium was then replaced by freshly prepared simulated intestinal 212 fluid (SIF) $\left(6.8 \mathrm{~g} \mathrm{~L}^{-1} \mathrm{KH}_{2} \mathrm{HPO}_{4}, 10 \mathrm{~g} \mathrm{~L}^{-1}\right.$ pancreatin) $\mathrm{pH} 7.5$ for an additional 22 hours.

213 Control experiments using SGF without pepsin for 2 hours followed by 22 hours in SIF 214 without pancreatin were run in parallel. Diclofenac is a weak acid $(\mathrm{pKa}=4)$ and thus 215 practically insoluble in acid. In order to measure the amount of the drug released in acidic 216 media, the media were neutralized at the end of the 2-hour test in SGF with or without pepsin 217 by adding $20 \mathrm{~mL}$ of $5 \mathrm{M} \mathrm{NaOH}$ and stirring using paddles for 5 minutes. Then a sample was withdrawn and filtered through $0.45 \mu \mathrm{m}$ filters prior to analysis for diclofenac sodium by high-performance liquid chromatography (HPLC; Knauer, Leeds, UK). In SIF with pancreatin, ten-mL samples were removed at predetermined times over a 22-hour period, centrifuged at $13,000 \mathrm{rpm}$ for 30 minutes, and filtered through $0.45 \mu \mathrm{m}$ filters prior to analysis for diclofenac sodium by high-performance liquid chromatography (HPLC; Knauer, Leeds, UK), using the same method described in Section 2.2.2.

\subsubsection{Scanning Electron Microscopy (SEM)}

The surface morphology of zein extrudates prior and after testing in media was studied using SEM. Samples were mounted onto stubs using double-sided tape and were platinum coated 
imaging process was performed in a high vacuum environment. Imaging process was performed with a FEI Inspect F50 SEM (Netherlands), mounted with a tungsten filament with an acceleration voltage of $1-30 \mathrm{kV}$.

\section{Results}

DSC and XRPD studies were engaged for determination of the physical state of the model drug, diclofenac sodium, in the prepared zein extrudates. Physical mixtures of DS and zein or granulated zein as well as DS and zein raw powders were used as controls.

241 DSC thermograms of DS and zein raw powders, physical mixtures of DS and zein powder or 242 granulated zein and zein extrudates of two different drug loadings are demonstrated in Figure 1. Zein powder showed a very broad endotherm with onset at $50{ }^{\circ} \mathrm{C}$ and peak temperature around $90{ }^{\circ} \mathrm{C}$. This can be associated with water loss (Tillekeratne and Easteal, 2000). The glass transition temperature of zein was observed at $168.4{ }^{\circ} \mathrm{C}$ which is in agreement with previous reports (Doğan Atik et al., 2008; Tillekeratne and Easteal, 2000). After $255^{\circ} \mathrm{C}$, the degradation of zein started with an exothermic peak coupled with an endothermic peak at 300 ${ }^{\circ} \mathrm{C}$ that has been attributed to thermal denaturation of zein through the breakdown of hydrogen bonds, electrostatic interactions, and dipole-dipole interactions and subsequent loss of tertiary protein structure (Gaona-Sánchez et al., 2015; Tillekeratne and Easteal, 2000). Beyond $330^{\circ} \mathrm{C}$, the exothermic degradation prevailed.

253 The glass transition temperature of zein was still evident in the physical mixture of zein and DS (PM_Zein-DS 12.5\%), however, it disappeared upon the incorporation of PEG used in the 
granulation of zein in PM_Granulated zein-DS $12.5 \%$. PEG acts as a plasticizer reducing the glass transition temperature of zein and making it difficult to be determined as it is shifted towards a lower temperature and is possibly overshadowed by the evaporative endotherm (Tillekeratne and Easteal, 2000). The onset of the thermal degradation peak of PM_Zein-DS $12.5 \%$ started at the same temperature as that of the zein alone sample $\left(\approx 255^{\circ} \mathrm{C}\right)$. However, the degradation of samples containing PEG 400 was seen at a lower temperature. This is possibly due to the thermal degradation of PEG 400 which starts at around $200{ }^{\circ} \mathrm{C}$, as shown in previous thermogravimetric analysis data (Phaechamud and Chitrattha, 2016).

The DSC thermogram of DS showed two endothermal events with a sharp peak at $53.44{ }^{\circ} \mathrm{C}$ and a broad one at $80.49{ }^{\circ} \mathrm{C}$ which correspond to the dehydration of DS tetrahydrate. A sharp endothermic melting peak at $287.55^{\circ} \mathrm{C}$ was observed and followed by decomposition (Bettini et al., 2004, 2000; Pasquali et al., 2007). The endothermic melting peak however disappeared in the DSC thermogram of PM_Zein-DS $12.5 \%$. This is because the thermal degradation of zein overshadowed the melting transition of the drug. Similarly, the drug peak was absent in all other samples containing drug and zein. Therefore, the overlap of zein and drug transitions precluded the determination of the physical state of the drug using DSC.

272

X-ray diffraction was used to analyze the degree of drug crystallinity in the studied samples.

274 As indicated by X-ray examinations (Figure 2), DS raw powder manifested the distinct peaks 275 of highly crystalline DS tetrahydrate, while there were no clear peaks in the X-ray diffractogram for the amorphous zein. The X-ray spectra of physical mixtures of zein and DS showed that the intensities of typical peaks for raw drug were lowered by the dilution effect of zein, particularly at low DS content (i.e. PM_Zein-DS 12.5\%). 
PM_Granulated zein-DS 12.5\%, unlike PM_Zein-DS 12.5\%, did not show any discernible

281 peaks and the diffraction pattern corresponded closely to that of amorphous zein. However,

282 the diffraction peaks of DS were seen in PM_Granulated zein-DS 37.5\% sample, albeit at 283 lower intensity compared to those observed with PM_Zein-DS 37.5\%. The reduction in the 284 crystallinity of the granulated samples containing PEG 400 could be explained by the 285 solubilisation of DS by PEG 400 (Khalil et al., 2000). These results suggest that the $12.5 \%$ of 286 DS was completely solubilized by PEG 400, whereas PEG 400 was saturated with DS at 37\% 287 drug loading and the remaining fraction of insolubilized drug contributed to the peaks 288 observed in the diffractogram of that sample.

290 Finally, the extruded samples (E_12.5_20; E_37.5_20) were characterized by the absence of the diffraction peaks in XRPD, signifying a drug amorphousization. The fact that amorphous pattern was obtained even at the highest DS content, i.e. $37.5 \%$ indicates that the extrusion process has higher capacity to amorphousize the drug compared to the granulation process.

3.2 In Vitro Drug Release Studies dissolution profiles of zein extrudates of four different drug loadings: $12.5 \%, 18.75 \%, 25 \%$ and $37.5 \% \mathrm{w} / \mathrm{w}$ in phosphate buffer $\mathrm{pH}$ 7.5. The higher the drug loading the faster was the drug release. Samples with the higher drug loadings, i.e. $25 \%$ and $37.5 \%$ showed almost immediate release profiles with circa $75 \%$ and $80 \%$ of drug being released in 1 hour, 
respectively. The drug release was the slowest from $12.5 \%$ DS loaded extrudates and

307 approached zero order release kinetics over the test duration, without initial burst release. The release profile from $18.75 \%$ DS loaded extrudates was biphasic with a rapid release of the

309 drug easily accessible at the surface of the extrudates and a slower second phase where

310 diffusion occurs. Bouman et al. (Bouman et al., 2015) have previously found that the drug 311 release from zein hot melt extruded injection moulded caplets was dependent on drug loading

312 in the case of ranitidine, but not in the case of paracetamol and indomethacin.

314 Zein is a swellable, nonerodable polymer, and drug release is thus expected to occur via

315 diffusion through pores between the polymeric chains or those created upon the dissolution of 316 the soluble components. In other words, increasing the drug $\%$ increases the soluble component, and simultaneously decreases the insoluble zein component, in the formulation, which upon dissolution creates more pores through which the drug can be released. In addition, the higher the concentration of the hydrophilic drug salt within the formulation, the greater is the overall hydrophilicity of the whole system. Higher hydrophilicity might translate into more rapid hydration (Bouman et al., 2016) and drug release. However, the differences in drug release could not be attributed to the physical state of the drug in the extrudates, as DS was present in the non-crystalline state at the lowest and highest drug loadings, as shown by the solid-state characterization discussed earlier.

\subsubsection{Effect of Buffer Concentration and Ionic Strength of Medium on Drug} Release

In addition to the standard compendial medium, i.e. $50 \mathrm{mM}$ phosphate buffer of $\mathrm{pH} 7.5$, diclofenac sodium release from zein extrudates was tested in media with different ionic strengths, i.e. in phosphate buffers of different buffer concentrations and in $50 \mathrm{mM}$ phosphate 
buffer with added amounts of $\mathrm{NaCl}$. These tests have been done because zein swelling is

333 known to be dependent on $\mathrm{pH}$ and ionic strength of the medium. Formulation E_12.5_20 was

334 selected for the studies described in this section. This is because this formulation exhibited a

335 controlled release behaviour, and it is therefore more likely to be discriminative, compared to

336 the other formulations.

337

338 Figure 4 compares the dissolution profiles of DS from the extrudates in phosphate buffers

$339(\mathrm{pH} 7.5)$ of different buffer concentrations (50, 100 and $200 \mathrm{mM}$ ). Water (used as a 340 reference), 50, 100, and $200 \mathrm{mM}$ PB have approximately ionic strength values of $0,90,180$,

$341360 \mathrm{mM}$, respectively. This range of ionic strength of the medium has been used to simulate

342 the effect of gastro-intestinal fed and fasted states on drug release from extended release

343 matrices (Asare-Addo et al., 2013a, 2013b). An increase in the buffer concentration from 0 to

$344200 \mathrm{mM}$ resulted in slower drug release. This result is in agreement with Berardi et al.

345 (Berardi et al., 2017b) where drug release from zein filled into hard gelatin capsules was

346 inversely dependent on PB concentration. The authors related the differences in drug release

347 in phosphate buffers of different ionic strengths to hydration/ swelling of zein protein.

348 Swelling of zein was found to be inversely proportional to the ionic strength of the medium.

349 Thus, the greater swelling at lower ionic strengths increased the porosity of the matrix, i.e.

350 the number and/or size of the channels, and ultimately increased the drug release. However,

351 in this study it can be observed that the release in water became slower than that in $50 \mathrm{mM}$

352 PB after 8 hours. This is most likely due to the lack of buffer capacity of water and the

353 subsequent decrease in DS solubility in water as more acid drug dissolves into the medium.

355 Human intestinal fluids contain bicarbonate buffer rather than phosphate buffer (Sheng et al., 356 2009). Moreover, the buffering capacity in vivo has been found to be much inferior to that of 
$50 \mathrm{mM}$ phosphate buffers (Hens et al., 2017; Tsume et al., 2012). This means that drugs, especially BCS class II weak acid drugs, could exhibit faster dissolution in vitro than in vivo, due to the higher $\mathrm{pH}$ and buffer capacity in vitro. Indeed, it has been calculated that the buffer capacity of bicarbonate buffers in vivo is equivalent to that of $8-45 \mathrm{mM}$ phosphate buffers

361 (Tsume et al., 2012). Further studies have shown that even lower phosphate buffer concentrations $(1-25 \mathrm{mM})$ are usually more biorelevant in simulating the influence of bicarbonate buffers on the dissolution of weak acid drugs (Krieg et al., 2015). We can thus speculate that the slower release of diclofenac sodium in water compared to $50 \mathrm{mM}$ PB might also occur, although to a lower extent, in vivo, where the buffer capacity is expected to be intermediate between the two media (i.e. water and $50 \mathrm{mM} \mathrm{PB}$ ) investigated here.

Next, we tested the effect of addition of $\mathrm{NaCl}$ into phosphate buffer on drug release. Figure 5 shows the drug release of DS from the extrudates in media containing increasing concentrations of salt. It can be observed that the addition of $0.1 \mathrm{M} \mathrm{NaCl}$ (ionic strength 190 $\mathrm{mM}$ ) in the buffer slowed down the drug release. However, no further decrease in drug release was obtained as the amount of $\mathrm{NaCl}$ was increased to $0.2 \mathrm{M}$. The obtained results are qualitatively and quantitatively similar to those of Figure 4. Again here, the decrease in drug release at increasing ionic strengths could be attributed to reduced swelling of zein matrices (Berardi et al., 2017b).

Performing a dissolution test with a two hours pre-incubation in SGF (without enzymes), followed by a switch to SIF (without enzymes) for the remaining time enables to simulate the effect of the gastric acid on the performance of the drug delivery system (Berardi et al., 
are known to swell more in acidic compared to more basic environments (Berardi et al., 2017a; Bouman et al., 2016, 2015). DS $(\mathrm{pKa}=4)$ is practically insoluble at $\mathrm{pH} \approx 1$ of the simulated gastric fluids (SGF) (Kincl et al., 2004). The low solubility precludes the drug diffusion from the dosage form. Indeed, the drug release in SGF was lower than in PB during the first two hours (Figure S1). Thereafter, the profiles in SIF and PB became rapidly superimposable, indicating that the pre-treatment in acid did not compromise the ability of the zein matrix to control the drug release. This is probably because, the preferential swelling of zein in acid is thought to be reversible (Berardi et al., 2018, 2017a).

392 Next, we studied the influence of the digestive enzymes on the drug release. Understanding the effect of proteases on the delivery system is of foremost important, given that zein is a protein. For this purpose, we compared the drug dissolution in SGF, followed by SIF either in presence or in absence of digestive enzymes, i.e. pepsin and pancreatin, respectively. Results are shown in Figure 6: digestive enzymes had a significant influence on the drug release. In the first two hours in SGF the drug release was only minimally affected by the presence of pepsin. However, upon switching to SIF, the drug release was much faster in the presence rather than in the absence of pancreatin, with circa $86 \%$ and $33 \%$ of the drug being released after a 12 hour-test duration, respectively.

401

To investigate this further, we took optical and SEM images of the extrudates before 403 incubation (Figure 7A) and after incubation in SGF (Figure 7B), SIF (Figure 7C) and SGF 404 followed by SIF (Figure 7D) both in presence and absence of enzymes. A comparison of the 405 images in Figure 7A and B reveals that the extrudates remained smooth both in the dry state 406 and upon incubation in SGF without pepsin. In the presence of pepsin, the surface appeared 407 slightly more striated and some superficial pores showed in the structure. However, the 
structural changes were microscopic and just on the surface. In contrast, the presence of pancreatin in SIF resulted in major morphological changes (Figure 7C): the structure of extrudates became an open network of pores visible both at the macroscopic and microscopic

411 levels, while it was solid in the absence of pancreatin. This indicates that pancreatin eroded 412 the zein-matrix by digestion. Figure 7D shows that the combined effect of pepsin and 413 pancreatin led to the formation of large and small pores in the structure. However, the erosion 414 was less than that observed with pancreatin alone (Figure 7C). We can hypothesise that the lesser digestion of zein matrix in SGF+SIF compared to SIF is due to an initial swelling and coalescence of the matrix in SGF, which partially impeded the following digestive action of the pancreatin in SIF.

419 Overall, these findings indicate that zein extrudates were much more affected by the enzymatic digestion by the intestinal proteases than by the gastric pepsin. In agreement with our study, several other authors, although testing different dosage forms, have shown that zein is more sensitive to pancreatin than pepsin (Alqahtani et al., 2017; Fu et al., 2002; Hu et al., 2016; Hurtado-López and Murdan, 2006; Kanig and Goodman, 1962). Despite these studies, there still is a question mark on whether zein-based dosage forms are affected by the digestive enzymes or not. It has been suggested that a univocal answer cannot be given, as this depends on the type of dosage form and manufacturing process and it should be evaluated on a case by case (Berardi et al., 2018). Our extrudates were sensitive to digestion, yet the digestion only sped up the drug release without causing a sudden and unwanted drug release burst. In other words, the enzymes partially digested zein, causing a modulation of the drug release, but did not abolish the ability of zein matrix to control the drug release. 
437 In this study, diclofenac sodium loaded zein based hot- melt extrudates were successfully produced. The drug was present in the non-crystalline state in zein extrudates at all drug loadings investigated. Drug loading as well as media parameters including ionic strength and presence of digestive enzymes influence the drug release rate were investigated. The results

441 indicate that the extent of the controlled release is drug loading dependent. A nearly zeroorder release with no burst release was obtained at the lowest drug loading $(12.5 \% \mathrm{w} / \mathrm{w})$.

Drug release rate can be further reduced by increasing ionic strength of medium, highlighting the potential influence of ionic strength in the gastro-intestinal tract on drug release from zein-based formulations. The presence of the digestive enzyme, namely pancreatin in simulated intestinal fluid significantly increased the drug release from zein extrudates by creating more pores through which the drug can diffuse. These results confirm the possibility of using zein as excipient in hot-melt extrusion for producing controlled release dosage forms.

453 The authors would like to acknowledge the technical support of Mr. Wadah Mahmoud (The 454 University of Jordan) for conducting the scanning electron microscopy experiments, Mr. 455 Yousef Abu-Salha (The University of Jordan) for conducting the XRPD analysis, Dr. Shadi 456 Madieh and Mr. Mohammad Abdulaziz (MSPharma, Amman, Jordan) for conducting the 457 DSC analysis. The authors would also like to thank The University of Jordan for the financial 458 support. 
Alqahtani, M.S., Islam, M.S., Podaralla, S., Kaushik, R.S., Reineke, J., Woyengo, T., Perumal, O., 2017. Food Protein Based Core-Shell Nanocarriers for Oral Drug Delivery: Effect of Shell Composition on in Vitro and in Vivo Functional Performance of Zein Nanocarriers. Mol. Pharm. 14, 757-769.

Andrews, G.P., Jones, D.S., Diak, O.A., McCoy, C.P., Watts, A.B., McGinity, J.W., 2008. The manufacture and characterisation of hot-melt extruded enteric tablets. Eur. J. Pharm. Biopharm. 69, 264-273. https://doi.org/https://doi.org/10.1016/j.ejpb.2007.11.001

Asare-Addo, K., Conway, B.R., Larhrib, H., Levina, M., Rajabi-Siahboomi, A.R., Tetteh, J., Boateng, J., Nokhodchi, A., 2013a. The effect of $\mathrm{pH}$ and ionic strength of dissolution media on in-vitro release of two model drugs of different solubilities from HPMC matrices. Colloids Surfaces B Biointerfaces 111, 384-391. https://doi.org/https://doi.org/10.1016/j.colsurfb.2013.06.034

Asare-Addo, K., Kaialy, W., Levina, M., Rajabi-Siahboomi, A., Ghori, M.U., Supuk, E., Laity, P.R., Conway, B.R., Nokhodchi, A., 2013b. The influence of agitation sequence and ionic strength on in vitro drug release from hypromellose (E4M and K4M) ER matrices - The use of the USP III apparatus. Colloids Surfaces B Biointerfaces 104, 54 60. https://doi.org/https://doi.org/10.1016/j.colsurfb.2012.11.020

Beck, M.I., Tomka, I., Waysek, E., 1996. Physico-chemical characterization of zein as a film coating polymer: A direct comparison with ethyl cellulose. Int. J. Pharm. 141, 137-150. https://doi.org/https://doi.org/10.1016/0378-5173(96)04630-3

Berardi, A., Bisharat, L., AlKhatib, H.S., Cespi, M., 2018. Zein as a Pharmaceutical Excipient in Oral Solid Dosage Forms: State of the Art and Future Perspectives. AAPS PharmSciTech 19, 2009-2022. https://doi.org/10.1208/s12249-018-1035-y

Berardi, A., Bisharat, L., Bonacucina, G., Casettari, L., Logrippo, S., Cespi, M., AlKhatib, H.S., Palmieri, G.F., 2017a. Formulation, swelling and dissolution kinetics study of zein based matrix tablets. Powder Technol. 310, 241-249. https://doi.org/https://doi.org/10.1016/j.powtec.2017.01.038

Berardi, A., Bisharat, L., Cespi, M., Basheti, I.A., Bonacucina, G., Pavoni, L., AlKhatib, H.S., 2017b. Controlled release properties of zein powder filled into hard gelatin capsules. Powder Technol. 320, 703-713. https://doi.org/https://doi.org/10.1016/j.powtec.2017.07.093

Bettini, R., Bertolini, G., Frigo, E., Rossi, A., Casini, I., Pasquali, I., Giordano, F., 2004. Interaction of pharmaceutical hydrates with supercritical CO2. J. Therm. Anal. Calorim. 
502

503

504

505

506

507

508

509

510

511

512

513

514

515

516

517

518

519

520

521

522

523

524

525

526

527

528

529

530

531

532

533

534

535

536

537

538

539

540

541

Bettini, R., Giordano, F., Donini, C., Massimo, G., Catellani, P.L., Colombo, P., 2000. Swelling force development as a result of hydrate formation in diclofenac sodium or nitrofurantoin tablets. STP Pharma Sci. 10, 335-340.

Bouman, J., Belton, P., Venema, P., Van Der Linden, E., De Vries, R., Qi, S., 2016. Controlled Release from Zein Matrices: Interplay of Drug Hydrophobicity and $\mathrm{pH}$. Pharm. Res. 33, 673-685. https://doi.org/10.1007/s11095-015-1818-8

Bouman, J., Belton, P., Venema, P., Van Der Linden, E., De Vries, R., Qi, S., 2015. The development of direct extrusion-injection moulded zein matrices as novel oral controlled drug delivery systems. Pharm. Res. 32, 2775-2786. https://doi.org/10.1007/s11095-0151663-9

Cabra, V., Arreguin, R., Vazquez-Duhalt, R., Farres, A., 2006. Effect of temperature and pH on the secondary structure and processes of oligomerization of $19 \mathrm{kDa}$ alpha-zein. Biochim. Biophys. Acta - Proteins Proteomics. https://doi.org/10.1016/j.bbapap.2006.04.002

Chuasuwan, B., Binjesoh, V., Polli, J.E., Zhang, H., Amidon, G.L., Junginger, H.E., Midha, K.K., Shah, V.P., Stavchansky, S., Dressman, J.B., Barends, D.M., 2009. Biowaiver Monographs for Immediate Release Solid Oral Dosage Forms: Diclofenac Sodium and Diclofenac Potassium. J. Pharm. Sci. 98, 1206-1219. https://doi.org/https://doi.org/10.1002/jps.21525

Corti, G., Cirri, M., Maestrelli, F., Mennini, N., Mura, P., 2008. Sustained-release matrix tablets of metformin hydrochloride in combination with triacetyl- $\beta$-cyclodextrin. Eur. J. Pharm. Biopharm. 68, 303-309. https://doi.org/https://doi.org/10.1016/j.ejpb.2007.06.004

Crowley, M.M., Zhang, F., Repka, M.A., Thumma, S., Upadhye, S.B., Kumar Battu, S., McGinity, J.W., Martin, C., 2007. Pharmaceutical Applications of Hot-Melt Extrusion: Part I. Drug Dev. Ind. Pharm. 33, 909-926. https://doi.org/10.1080/03639040701498759

Doğan Atik, İ., Özen, B., T1hmınlıŏlu, F., 2008. Water vapour barrier performance of cornzein coated polypropylene (PP) packaging films. J. Therm. Anal. Calorim. 94, 687-693. https://doi.org/10.1007/s10973-008-9360-0

Esen, A., 1986. Separation of Alcohol-Soluble Proteins (Zeins) from Maize into Three Fractions by Differential Solubility. Plant Physiol. 80, 623-627.

Fu, T.-J., Abbott, U.R., Hatzos, C., 2002. Digestibility of Food Allergens and Nonallergenic Proteins in Simulated Gastric Fluid and Simulated Intestinal FluidA Comparative Study. J. Agric. Food Chem. 50, 7154-7160. https://doi.org/10.1021/jf020599h

Gaona-Sánchez, V.A., Calderón-Domínguez, G., Morales-Sánchez, E., Chanona-Pérez, J.J., Velázquez-de la Cruz, G., Méndez-Méndez, J. V, Terrés-Rojas, E., Farrera-Rebollo, R.R., 2015. Preparation and characterisation of zein films obtained by electrospraying. Food Hydrocoll. 49, 1-10. https://doi.org/https://doi.org/10.1016/j.foodhyd.2015.03.003

Georget, D.M.R., Barker, S.A., Belton, P.S., 2008. A study on maize proteins as a potential new tablet excipient. Eur. J. Pharm. Biopharm. 
Hens, B., Tsume, Y., Bermejo, M., Paixao, P., Koenigsknecht, M.J., Baker, J.R., Hasler, W.L., Lionberger, R., Fan, J., Dickens, J., Shedden, K., Wen, B., Wysocki, J., Loebenberg, R., Lee, A., Frances, A., Amidon, G., Yu, A., Benninghoff, G., Salehi, N., Talattof, A., Sun, D., Amidon, G.L., 2017. Low Buffer Capacity and Alternating Motility along the Human Gastrointestinal Tract: Implications for in Vivo Dissolution and Absorption of Ionizable Drugs. Mol. Pharm. 14, 4281-4294. https://doi.org/10.1021/acs.molpharmaceut.7b00426

Hu, S., Wang, T., Fernandez, M.L., Luo, Y., 2016. Development of tannic acid cross-linked hollow zein nanoparticles as potential oral delivery vehicles for curcumin. Food Hydrocoll. 61, 821-831. https://doi.org/https://doi.org/10.1016/j.foodhyd.2016.07.006

Hurtado-López, P., Murdan, S., 2006. Zein microspheres as drug/antigen carriers: A study of their degradation and erosion, in the presence and absence of enzymes. J. Microencapsul. 23, 303-314. https://doi.org/10.1080/02652040500444149

Jantratid, E., De Maio, V., Ronda, E., Mattavelli, V., Vertzoni, M., Dressman, J.B., 2009. Application of biorelevant dissolution tests to the prediction of in vivo performance of diclofenac sodium from an oral modified-release pellet dosage form. Eur. J. Pharm. Sci. 37, 434-441. https://doi.org/https://doi.org/10.1016/j.ejps.2009.03.015

Kanig, J.L., Goodman, H., 1962. Evaluative Procedures for Film-Forming Materials Used in Pharmaceutical Applications. J. Pharm. Sci. 51, 77-83. https://doi.org/https://doi.org/10.1002/jps.2600510115

Katayama, H., Kanke, M., 1992. Drug release from directly compressed tablets containing zein. Drug Dev. Ind. Pharm. 18, 2173-2184. https://doi.org/10.3109/03639049209038755

Khalil, E., Najjar, S., Sallam, A., 2000. Aqueous Solubility of Diclofenac Diethylamine in the Presence of Pharmaceutical Additives: A Comparative Study with Diclofenac Sodium. Drug Dev. Ind. Pharm. 26, 375-381. https://doi.org/10.1081/DDC-100101243

Kincl, M., Meleh, M., Veber, M., Vrecer, F., 2004. Study of physicochemical parameters affecting the release of diclofenac sodium from lipophilic matrix tablets. Acta Chim. Slov. 51, 409-425.

Krieg, B.J., Taghavi, S.M., Amidon, G.L., Amidon, G.E., 2015. <em>In Vivo</em> Predictive Dissolution: Comparing the Effect of Bicarbonate and Phosphate Buffer on the Dissolution of Weak Acids and Weak Bases. J. Pharm. Sci. 104, 2894-2904. https://doi.org/10.1002/jps.24460

Lawton, J.W., 2002. Zein: A History of Processing and Use. Cereal Chem. 79, 1-18. https://doi.org/doi:10.1094/CCHEM.2002.79.1.1

Li, X.N., Guo, H.X., Heinamaki, J., 2010. Aqueous coating dispersion (pseudolatex) of zein improves formulation of sustained-release tablets containing very water-soluble drug. J. Colloid Interface Sci. 345, 46-53. https://doi.org/10.1016/j.jcis.2010.01.029

O’Donnell, P.B., Wu, C., Wang, J., Wang, L., Oshlack, B., Chasin, M., Bodmeier, R., McGinity, J.W., 1997. Aqueous pseudolatex of zein for film coating of solid dosage 
605

606

607

608

609

610

611

612

613

614

615

616

617

618

619

620

forms. Eur. J. Pharm. Biopharm. 43, 83-89. https://doi.org/https://doi.org/10.1016/S0939-6411(96)00013-6

Pasquali, I., Bettini, R., Giordano, F., 2007. Thermal behaviour of diclofenac, diclofenac sodium and sodium bicarbonate compositions. J. Therm. Anal. Calorim. 90, 903-907. https://doi.org/10.1007/s10973-006-8182-1

Phaechamud, T., Chitrattha, S., 2016. Pore formation mechanism of porous poly(dl-lactic acid) matrix membrane. Mater. Sci. Eng. C 61, 744-752. https://doi.org/https://doi.org/10.1016/j.msec.2016.01.014

Rani, M., Mishra, B., 2004. Comparative in vitro and in vivo evaluation of matrix, osmotic matrix, and osmotic pump tablets for controlled delivery of diclofenac sodium. AAPS PharmSciTech 5, 153-159. https://doi.org/10.1208/pt050471

Raza, A., Hayat, U., Wang, H.-J., Wang, J.-Y., 2020. Preparation and evaluation of captopril loaded gastro-retentive zein based porous floating tablets. Int. J. Pharm. 119185. https://doi.org/https://doi.org/10.1016/j.ijpharm.2020.119185

Raza, A., Shen, N., Li, J., Chen, Y., Wang, J.-Y., 2019. Formulation of zein based compression coated floating tablets for enhanced gastric retention and tunable drug release. Eur. J. Pharm. Sci. 132, 163-173. https://doi.org/10.1016/j.ejps.2019.01.025

Selling, G.W., 2010. The effect of extrusion processing on Zein. Polym. Degrad. Stab. 95, 2241-2249. https://doi.org/10.1016/j.polymdegradstab.2010.09.013

Sheng, J.J., McNamara, D.P., Amidon, G.L., 2009. Toward an In Vivo Dissolution Methodology: A Comparison of Phosphate and Bicarbonate Buffers. Mol. Pharm. 6, 2939. https://doi.org/10.1021/mp800148u

Shukla, R., Cheryan, M., 2001. Zein: the industrial protein from corn. Ind. Crops Prod. 13, 171-192. https://doi.org/https://doi.org/10.1016/S0926-6690(00)00064-9

Tillekeratne, M., Easteal, A.J., 2000. Modification of zein films by incorporation of poly (ethylene glycol) s. Polym. Int. 49, 127-134.

Tsume, Y., Langguth, P., Garcia-Arieta, A., Amidon, G.L., 2012. In silico prediction of drug dissolution and absorption with variation in intestinal $\mathrm{pH}$ for BCS class II weak acid drugs: ibuprofen and ketoprofen. Biopharm. Drug Dispos. 33, 366-377. https://doi.org/10.1002/bdd.1800

Wang, Q., Yin, L., Padua, G.W., 2008. Effect of hydrophilic and lipophilic compounds on zein microstructures. Food Biophys. 3, 174-181. https://doi.org/10.1007/s11483-0089080-9

Young, C.R., Koleng, J.J., McGinity, J.W., 2002. Production of spherical pellets by a hotmelt extrusion and spheronization process. Int. J. Pharm. 242, 87-92. https://doi.org/https://doi.org/10.1016/S0378-5173(02)00152-7 